\title{
A testbed for long-range LoRa communication
}

\section{Other Conference Item}

Author(s):

Trüb, Roman (1); Da Forno, Reto; Gsell, Tonio; Beutel, Jan (1); Thiele, Lothar

Publication date:

2019-04-16

Permanent link:

https://doi.org/10.3929/ethz-b-000338740

Rights / license:

In Copyright - Non-Commercial Use Permitted

Originally published in:

https://doi.org/10.1145/3302506.3312484 


\title{
Demo Abstract: A Testbed for Long-Range LoRa Communication
}

\author{
Roman Trüb, Reto Da Forno, Tonio Gsell, Jan Beutel, Lothar Thiele \\ Computer Engineering and Networks Laboratory, ETH Zurich \\ Zurich, Switzerland \\ rtrueb@ethz.ch
}

\begin{abstract}
Designing and testing low-power wireless communication protocols often requires experimental deployments on real hardware in realistic settings. Infrastructure testbeds have the advantage that they allow reproducible results using different network configurations. However, most testbeds are either in- or outdoor only and do not span long and short ranges at the same time. In this work, we present an extension to the popular FlockLab testbed on a campus-scale in order to better support testing of long-range communciation, for example using the LoRa modulation. Different to existing LoRa test networks where specific protocol layers are fixed, we support custom modification above the physical hardware (above PHY) which allows the development and testing of alternative full custom MAC layers that are not based on LoRaWAN.
\end{abstract}

\section{CCS CONCEPTS}

- Networks $\rightarrow$ Wide area networks; Sensor networks.

\section{KEYWORDS}

LoRa, long-range, testbed, FlockLab, time synchronization

\section{ACM Reference Format:}

Roman Trüb, Reto Da Forno, Tonio Gsell, Jan Beutel, Lothar Thiele. 2019. Demo Abstract: A Testbed for Long-Range LoRa Communication. In The 18th International Conference on Information Processing in Sensor Networks (co-located with CPS-IoT Week 2019) (IPSN '19), April 16-18, 2019, Montreal, QC, Canada. ACM, New York, NY, USA, 2 pages. https://doi.org/10.1145/ 3302506.3312484

\section{MOTIVATION}

Testbeds for wireless communication protocols are useful when developing and testing protocols since it reduces the effort to deploy test networks repeatedly [10]. Furthermore, such a testbed improves the reproducibility of experiments and allows to share infrastructure [5]. Contrary to many other testbeds, FlockLab [2, 6] has always supported a mix of in- and outdoor placements. However, all nodes were located in rather close proximity to each other in and around an office building. This setup has been vastly popular: the FlockLab testbed has been operated since 2012, run over 53822 tests by 325 distinct users and reached an average annual utilization of over 55\%. In recent years, long-range communication for IoT applications has become increasingly important. Although publicly accessible test networks for long-range protocols exist (e.g. e.g. TheThingsNetwork[3] for LoRa), they are only of limited use for designing and testing on the lower layers since modifications of the fixed MAC layer implementation are typically not possible. To the best of our knowledge, sensor network testbeds that support both long-range communication and and provide the option to monitor and control communication layers above the physical hardware do not exist. Therefore, we are currently extending the existing short baseline distances in FlockLab by adding additional nodes on rooftop locations and with significantly larger spacing. The vision is to extend FlockLab to span the whole campus $(\leq 1 \mathrm{~km}$ link distance) or even parts of the city $(\sim 5-10 \mathrm{~km})$. Similar efforts have been made for $802.11 \mathrm{~b} / \mathrm{g}$ mesh network research at MIT with the RoofNet [4].

\section{FLOCKLAB SYSTEM SETUP}

The FlockLab testbed consists of observer nodes and a backend server. Each observer node can host 4 targets (devices under test), that are modules with radio and microcontroller chips used for wireless protocol development. The observer provides the infrastructure to power, program, stimulate, log and profile the targets. When running a test on FlockLab, the server instructs the observers to setup and start the targets. While the test is running, the observers independently collect profiling data which they aggregate and send to the server once the test is finished. Users can then access all test data which is stored in a database on the server.

Topology: Currently, the extended FlockLab testbed consists of 28 nodes located on a single floor inside an office building $(75 \times 35 \mathrm{~m})$ and 2 rooftop nodes located up to $470 \mathrm{~m}$ away (see Figure 1).

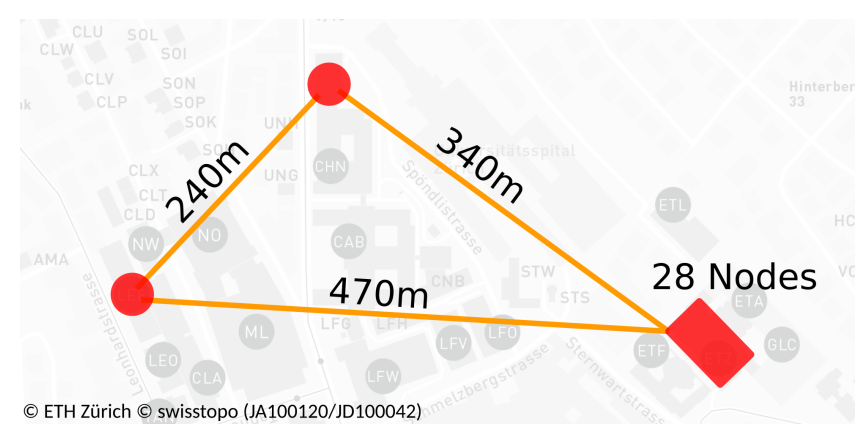

Figure 1: FlockLab testbed extension to rooftop nodes.

Services and Time Synchronization: For designing and testing low-power wireless network protocols, FlockLab offers the services listed in Table 1. The GPIO services provides access to up to 5 GPIO pins of the target. This allows to observe or initiate state changes or measure duty-cycles with high accuracy. The power profiling allows to perform fine-grained power measurements. Serial output of the target is logged by the serial tracing service. Interaction via the serial interface is supported by the serial forwarder service. Both the GPIO and the power profiling services provide high temporal accuracy which is required for developing low-power wireless protocols on the lower layers of the network stack.

The time synchronization of the original FlockLab testbed uses a custom wireless time synchronization protocol based on Glossy [7] 


\begin{tabular}{lll}
\hline FlockLab Service & Max. Rate & Time Sync \\
\hline GPIO tracing & $10 \mathrm{MHz}$ & GlossySync/GPS \\
GPIO actuation & $10 \mathrm{MHz}$ & GlossySync/GPS \\
Power profiling & $28 \mathrm{ksamples} / \mathrm{s}$ & GlossySync/GPS \\
Serial tracing & & NTP \\
Serial forwarding & & -
\end{tabular}

$\overline{\text { Table 1: FlockLab requires tight time synchronization. }}$

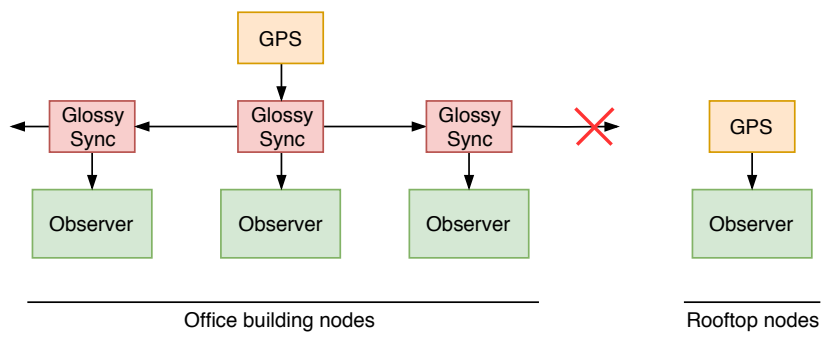

Figure 2: Heterogeneous time synchronization architecture.

\begin{tabular}{lll}
\hline & Mean & Std dev. \\
\hline GlossySync - GlossySync & $0.016 \mu \mathrm{s}$ & $0.202 \mu \mathrm{s}$ \\
GPS - GlossySync & $0.692 \mu \mathrm{s}$ & $0.206 \mu \mathrm{s}$ \\
GPS - GPS & $0.050 \mu \mathrm{s}$ & $0.115 \mu \mathrm{s}$
\end{tabular}

Table 2: FlockLab time synchronization performance.

(GlossySync) referenced to a 1 Pulse-per-second (PPS) signal sourced from a ublox LEA-6T GPS receiver (see left part of Figure 2). This provides an offset between two observers below $1 \mu$ s which is sufficient for most applications. However, recent work incorporating the time-of-flight of radio signals has shown that higher accuracy is required [8] and FlockLab has been outfitted temporarily with extra GPS receivers in order to perform these tests. This experience and the fact that it is not possible to extend the currently used short-range GlossySync time synchronization required a different approach. The option to use PTP over Ethernet [1] (accuracy $<1 \mu \mathrm{s}$ ) has been explored but not implemented since it would require specialized hardware and infrastructure on the networking segment. Therefore, each rooftop node is equipped with a dedicated GPS receiver providing a 1 PPS signal directly to the FlockLab observer. Since the used reference timebase (GPS) is the same for both systems, we only need to make sure that the offset between GlossySync and GPS is properly compensated. Measurements of the resulting time difference when timestamping the same GPIO event (hard wired) between two observers using different time synchronization methods are listed in Table 2.

Antenna Options: Since FlockLab supports multiple independent target devices on one observer, it is possible to install several instances of the same target hardware using different antennas (lowvs. high-gain) without the need for complex RF switching or cabling. This allows to select different antennas for individual tests.

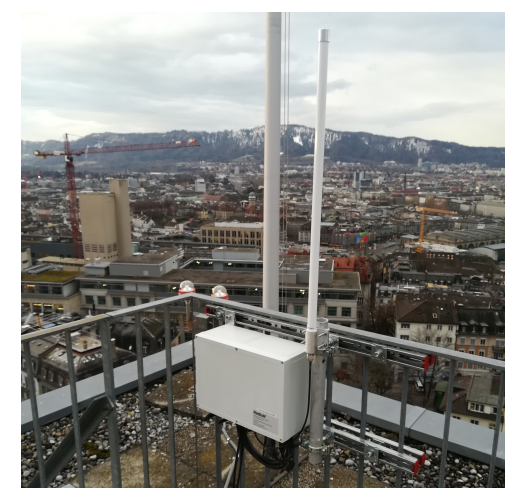

Figure 3: Rooftop node of FlockLab with high-gain antenna.

\section{DEMONSTRATION SETUP}

In this demonstration, we will show the FlockLab hardware setup (observer with targets) as well as demonstrate running tests on both short-range radio architectures (TinyNode, TmoteSky, DPP2CC430) as well as long-range radio architectures (DPP2-SX1262). Furthermore, a visualization of the resulting GPIO traces can be observed. The DPP2-CC430 and DPP-SX1262 are based on the Dual Processor Platform $\left(\mathrm{DPP}^{1}\right)$ [9].

\section{REFERENCES}

[1] 2008. IEEE Standard for a Precision Clock Synchronization Protocol for Networked Measurement and Control Systems.

[2] FlockLab. https://www.flocklab.ethz.ch/. [acc. 14-Feb-2019].

[3] The Things Network. https://www.thethingsnetwork.org/. [acc. 14-Feb-2019].

[4] Daniel Aguayo, John Bicket, Sanjit Biswas, and DD Couto. 2004. MIT Roofnet: Construction of a community wireless network. Technical Report. MIT Computer Science and Artificial Intelligence.

[5] Carlo A Boano, Simon Duquennoy, Anna Förster, Omprakash Gnawali, Romain Jacob, Hyung-Sin Kim, Olaf Landsiedel, Ramona Marfievici, Luca Mottola, Gian Pietro Picco, et al. 2018. IoTBench: Towards a Benchmark for Low-power Wireless Networking. In 1st Workshop on Benchmarking Cyber-Physical Networks and Systems (CPSBench 2018).

[6] Roman Lim, Federico Ferrari, Marco Zimmerling, Christoph Walser, Philipp Sommer, and Jan Beutel. 2013. FlockLab: a testbed for distributed, synchronized tracing and profiling of wireless embedded systems. In Proceedings of the 12th international conference on Information processing in sensor networks. ACM, 153166.

[7] Roman Lim, Balz Maag, Benjamin Dissler, Jan Beutel, and Lothar Thiele. 2015. A testbed for fine-grained tracing of time sensitive behavior in wireless sensor networks. In Local Computer Networks Conference Workshops (LCN Workshops), 2015 IEEE 40th. IEEE, 619-626.

[8] Roman Lim, Balz Maag, and Lothar Thiele. 2016. Time-of-Flight Aware Time Synchronization for Wireless Embedded Systems. In EWSN. 149-158.

[9] Felix Sutton, Marco Zimmerling, Reto Da Forno, Roman Lim, Tonio Gsell, Georgia Giannopoulou, Federico Ferrari, Jan Beutel, and Lothar Thiele. 2015. Bolt: A Stateful Processor Interconnect. In Proceedings of the 13th ACM Conference on Embedded Networked Sensor Systems. ACM, 267-280.

[10] Anne-Sophie Tonneau, Nathalie Mitton, and Julien Vandaele. 2014. A survey on (mobile) wireless sensor network experimentation testbeds. In International Conference on Distributed Computing in Sensor Systems (DCOSS). IEEE, 263-268.

\footnotetext{
${ }^{1}$ See a separate DPP-related demo submission to IPSN 2019.
} 